INPLASY

PROTOCOL

To cite: Cai et al. Prevalence of sleep disturbance in children and adolescents during COVID-19 pandemic: a metaanalysis and systematic review of epidemiological surveys. Inplasy protocol 202190098. doi:

10.37766/inplasy2021.9.0098

Received: 27 September 2021

Published: 27 September 2021

Corresponding author: Hong Cai

yc07640@mac.com

Author Affiliation:

University of Macau

Support: None.

Review Stage at time of this submission: Faculty of Health Sciences, University of Macau, Avenida da Universidade, Taipa.

Conflicts of interest: None declared.

\section{Prevalence of sleep disturbance in children and adolescents during COVID-19 pandemic: a meta-analysis and systematic review of epidemiological surveys}

Cai, $\mathrm{H}^{1}$; Chen, $\mathrm{P}^{2}$; Xiang, $\mathrm{YT}^{3}$.

Review question / Objective: Participants: Children and Adolescents; Intervention: not applicable (NA); Control: NA; Outcomes: the prevalence of sleep disturbances or data that could generate prevalence of sleep disturbances; and Study: epidemiological surveys.

Condition being studied: The indirect accompaniment of the COVID-19 pandemic such as lockdown, travel restriction and close of the school and online teaching have also influence the lifestyle in children and adolescent(Castagnoli et al., 2020; Mustafa and Selim, 2020). The effect on sleep disturbance in healthy children and adolescent and those with neurobehavioral disorders has been studied and the pooled prevalence of any sleep disturbance in COVID-19 pandemic was $54 \%(95 \% \mathrm{Cl}: 50-57 \%)$ (Sharma et al., 2021). However, this meta-analysis only included three original studies. Hence, the aim of this meta analysis was conducted a meta-analysis of the global prevalence of clinically elevated symptoms of sleep disturbance in child and adolecents during the COVID-19 pandemic.

INPLASY registration number: This protocol was registered with the International Platform of Registered Systematic Review and Meta-Analysis Protocols (INPLASY) on 27 September 2021 and was last updated on 27 September 2021 (registration number INPLASY202190098).

\section{INTRODUCTION}

Review question / Objective: Participants: Children and Adolescents; Intervention: not applicable (NA); Control: NA; Outcomes: the prevalence of sleep disturbances or data that could generate prevalence of sleep disturbances; and Study: epidemiological surveys.

Condition being studied: The indirect accompaniment of the COVID-19 pandemic such as lockdown, travel restriction and close of the school and online teaching 
have also influence the lifestyle in children and adolescent(Castagnoli et al., 2020; Mustafa and Selim, 2020). The effect on sleep disturbance in healthy children and adolescent and those with neurobehavioral disorders has been studied and the pooled prevalence of any sleep disturbance in COVID-19 pandemic was $54 \%(95 \% \mathrm{Cl}$ : $50-57 \%$ )(Sharma et al., 2021). However, this meta-analysis only included three original studies. Hence, the aim of this meta analysis was conducted a meta-analysis of the global prevalence of clinically elevated symptoms of sleep disturbance in child and adolecents during the COVID-19 pandemic.

\section{METHODS}

Search strategy: Two investigators (HC and PC) independently searched the literature in PubMed, PsycINFO, Web of Science and two Chinese database (Chinese Nation knowledge Infrastructure (CNKI) and WANFANG) from their 01 January 2020 until 20 September 2021, using the following terms: (Sleep Initiation and Maintenance Disorders [MeSH Terms] OR sleep disturbance OR insomnia OR sleep problem OR sleep disorder OR sleep symptom OR sleep*) AND (adolescent [MeSH Terms] OR child OR children OR preschool OR pediatrics OR infants OR toddlers) AND (2019-ncov* OR 2019ncov* OR 2019n-cov* OR coronaviru* OR corona viru* OR covid OR covid-19 OR covid19* OR novel cov* OR ncov* OR covid-2019 OR covid2019 OR SARS-COV2* OR SARS COV-2* OR SARS COV2* OR SARS COV19 OR SARS COV-19 OR SARS-COV-2019 OR SARS COV 2019 OR SARS COV-2019 OR severe acute respiratory syndrome or severe acute respiratory disease) AND (epidemiology OR prevalence OR rate).

Participant or population: Children and Adolescents.

Intervention: Not applicable (NA).

Comparator: Not applicable (NA).

Study designs to be included: Epidemiological surveys.
Eligibility criteria: Participants: Children and Adolescents; Intervention: not applicable (NA); Control: NA; Outcomes: the prevalence of sleep disturbances or data that could generate prevalence of sleep disturbances; and Study: epidemiological surveys. When more than one paper was published based on the same dataset, only the one with the largest sample was included.

Information sources: Two investigators (HC and $P C$ ) independently searched the literature in PubMed, PsycINFO, Web of Science and two Chinese database (Chinese Nation knowledge Infrastructure (CNKI) and WANFANG) from their 01 January 2020 until 20 September 2021, using the following terms: (Sleep Initiation and Maintenance Disorders [MeSH Terms] OR sleep disturbance OR insomnia OR sleep problem OR sleep disorder OR sleep symptom OR sleep*) AND (adolescent [MeSH Terms] OR child OR children OR preschool OR pediatrics OR infants OR toddlers) AND (2019-ncov* OR 2019ncov* OR 2019n-cov* OR coronaviru* OR corona viru* OR covid OR covid-19 OR covid19* OR novel cov* OR ncov* OR covid-2019 OR covid2019 OR SARS-COV2* OR SARS COV-2* OR SARS COV2* OR SARS COV19 OR SARS COV-19 OR SARS-COV-2019 OR SARS COV 2019 OR SARS COV-2019 OR severe acute respiratory syndrome or severe acute respiratory disease) AND (epidemiology OR prevalence OR rate). Two investigators (HC and PC) independently screened the titles and abstracts, and then read the full texts of relevant papers for eligibility. Moreover, reference lists of relevant reviews were checked manually to identify potentially missing studies.

Main outcome(s): The prevalence of sleep disturbances or data that could generate prevalence of sleep disturbances.

Quality assessment / Risk of bias analysis: Study quality was assessed using a standardized instrument for epidemiological studies (Boyle, 1998b; Loney, 1998) with 8 items as follows: (1) Target population was defined clearly, (2) Probability sampling or entire population 
surveyed (3) Response rate was equal or greater than $80 \%$, (4) Non-responders clearly described (5) Sample representative of the target population (6) Data collection methods standardized (7) Validated criteria used to measure sleep disturbance (8) Prevalence estimates given with confidence intervals and detailed by subgroups (if applicable). The total score ranges from 0 to 8 . Studies with a total score of "7-8" were considered as "high quality", "4-6" as "moderate quality" and "0-3" as "low quality" (Yang,2016).

Strategy of data synthesis: The random effects model was used to calculate the pooled prevalence of sleep disturbance with their $95 \%$ confidence intervals $(95 \%$ Cls). The heterogeneity between studies was assessed with the 12 statistic, and $12>$ $50 \%$ was considered an indication of high heterogeneity (Higgins et al., 2003).

Subgroup analysis: The moderating effects of categorical variables (e.g., symptom report, child and/or adolescent and region was classified by broad WHO regional classification (Africa/Americas/Eastern Mediterranean/ Europe/South East Asia/ Western Pacific) (Chen et al., 2018)) and continuous variables (e.g., percentage of males, mean age and quality evaluation score) were examined using metaregression analyses, respectively.

Sensitivity analysis: Sensitivity analyses were performed to identify outlying studies by excluding studies one by one. Publication bias was estimated with funnel plots and Egger's test (Egger et al., 1997). A $p$ value $<0.05$ was considered statistically significant (two-tailed).

Country(ies) involved: Not reported.

Keywords: child, adolescent, sleep disturbance.

Contributions of each author:

Author 1 - Hong Cai.

Email: yc07640@mac.com

Author 2 - Pan Chen.

Email: yc17609@mac.com
Author 3 - Yu-tao Xiang. Email: xyutly@gmail.com 